\title{
APEXOGÉNESIS DE UN MOLAR INFERIOR PERMANENTE JOVEN CON MTA
}

\author{
Andrés Callejas Ospina ${ }^{*}$, Esp. $_{1}$, William Andrés Jaramillo Builes, est. odontología ${ }_{1}$
}

${ }_{1}$ Universidad Cooperativa de Colombia, sede Medellín, Colombia

Recibido: 7 de marzo del 2013. Aprobado: 30 de abril del 2013.

*Autor de correspondencia: Andrés Callejas, Facultad de Odontología, Universidad Cooperativa de Colombia, Medellín, Colombia, (57) 42706466 , Carrera 47 n. ${ }^{\circ} 37$ Sur-18, correo electrónico: jorge.callejas@campusucc.edu.co

Cómo citar este artículo: Callejas Ospina A, Jaramillo Builes WA. Apexogénesis de un molar inferior permanente joven con MTA. Revista Nacional de Odontología. 2013; 9(16): 93-97.

Resumen. Uno de los principales objetivos de la terapia endodóntica es realizar una limpieza químico-mecánica del sistema de conductos radiculares con la eliminación completa del tejido pulpar en dientes que tienen su desarrollo completo y han sufrido algún tipo de patología pulpar; sin embargo, hay casos en los que el objetivo es mantener parte de ese tejido para promover el desarrollo de las raíces en dientes que han sufrido algún tipo de afección y no han terminado aún su formación radicular. Para estos casos, la apexogénesis es un tratamiento que permite inducir el cierre apical de dientes con formación radicular incompleta. Este reporte de caso describe el tratamiento de una niña de 7 años de edad que consulta al Servicio de Urgencias de la Universidad Cooperativa de Colombia, sede Medellín, relatando dolor ocasional en la zona mandibular izquierda. Al examen clínico el diente afectado fue examinado clínica y radiográficamente; en el primer molar inferior permanente izquierdo se observó una caries extensa y ápices inmaduros. Se le realizó un procedimiento conservador de apexogénesis con мта y seguimiento a un año evidenciando el cierre apical de sus raíces totalmente asintomático.

Palabras clave: apexogénesis, ápice radicular inmaduro, dolor, hidróxido de calcio, MTA, pulpotomía.

\section{Apexogenesis of a First Permanent Mandibular Molar with мтA}

Abstract. One of the main goals of endodontic therapy is to chemically and mechanically clean the root canal system with the complete removal of the pulp tissue in teeth that are fully grown and have suffered some kind of pulp pathology. However, there are cases in which the goal is to keep some of that tissue to promote root development in teeth that have undergone some type of pathology and have not yet finished their root formation. For these cases, the apexogenesis is a treatment that can induce apical closure of teeth with incomplete root formation. This case report describes treatment of a 7 year old girl that consulted at the Department of Dental Emergencies of the Universidad Cooperativa de Colombia with an occasional pain on her left lower jaw. The tooth responsible was examined clinically and through $\mathrm{x}$-rays. On clinical examination, the first permanent left mandibular molar had extensive caries and immature apices. Radiographic examination revealed an immature root. We performed conservative treatment (apexogenesis) and then a 1 year follow up that evidenced apical closure and the disappearance of the symptoms.

Keywords: apexogenesis, immature tooth root apex, pain, calcium hydroxide, MTA, pulpotomy.

\section{Apexogênese de um molar inferior permanente jovem com MTA}

Resumo. Um dos principais objetivos da terapia endodôntica é realizar uma limpeza químico-mecânica do sistema de condutos radiculares com a eliminação completa do tecido pulpar em dentes que têm seu desenvolvimento completo e sofrem algum tipo de patologia pulpar; contudo, há casos nos quais o objetivo é manter parte desse tecido para promover o desenvolvimento das raízes em dentes que sofreram algum tipo de afecção e não terminaram ainda sua formação radicular. Para esses casos, a apexogênese é um tratamento que permite induzir o fechamento apical de dentes com formação radicular incompleta. Este relatório de caso descreve o tratamento de uma criança de sete anos que consulta o Pronto-socorro da Universidade Cooperativa da Colômbia, sede Medellín, relatando dor ocasional na zona mandibular esquerda. Ao exame clínico, o dente afetado foi examinado clínica e radiograficamente; no primeiro molar inferior permanente esquerdo, observou-se uma cárie extensa e ápices imaturos. Realizou-se nela um procedimento conservador de apexogênese com MTA e seguimento de um ano, o que evidenciou o fechamento apical de suas raízes totalmente assintomático.

Palavras-chave: apexogênese, ápice radicular imaturo, dor, hidróxido de cálcio, MTA, pulpotomia. 


\section{Introducción}

La apexogénesis se puede definir como un procedimiento que es realizado en pulpas vitales con el fin de promover el desarrollo fisiológico de las raíces de los dientes y el cierre de porción apical en dientes con desarrollo radicular incompleto y que, debido a caries profunda, traumatismo o fractura, presentan una exposición pulpar y no es posible realizar un procedimiento endodóntico convencional debido a que los ápices están abiertos [1]. Un diente al momento de erupcionar tiene desarrollado del 60 al $80 \%$ de su raíz, y generalmente es más susceptible a las exposiciones pulpares por caries, ya que los tejidos duros no se encuentran suficientemente mineralizados, permitiendo más rápido la difusión de los ácidos [2]; por tanto, se hace necesario en algunos casos llevar a cabo una terapia pulpar en estos dientes.

Entre los materiales más utilizados en los procedimientos de apexogénesis está el hidróxido de calcio $\left(\mathrm{Ca}(\mathrm{OH})_{2}\right)$, introducido por Hermann como un relleno biológico [3]. Sin embargo, su aparente éxito en la terapia pulpar vital ha sido cuestionado, ya que genera necrosis superficial del tejido pulpar cuando es colocado en contacto directo con este en su estado puro, gracias a su $\mathrm{pH}$ alcalino ( $\mathrm{pH} 12)$, siendo cáustico para el tejido pulpar [4]. Estudios han mostrado que el hidróxido de calcio es muy tóxico para las células, además de presentar un efecto de disolución del tejido, lo que hace que su uso en terapias pulpares sea cuestionado [5-8].

Otro material que actualmente ha sido muy utilizado en estos procedimientos es el mineral trióxido agregado, más comúnmente conocido como MTA. Este material provee un excelente capacidad de selle sobre la pulpa vital al no disolverse fácilmente cuando este se usa $[9,10]$. El material fue introducido en 1993 por Torabinejad, patentado en 1995 y aprobado para procedimientos endodónticos en 1998 [11-13]. El MTA es un polvo compuesto principalmente de silicato tricálcico, silicato dicálcico, aluminato dicálcico, sulfato dehidrato de calcio y óxido de bismuto. Este material al ser hidratado se convierte en un gel coloidal que se solidifica en aproximadamente 3 a 4 horas $[14,15]$.

El MTA ha reportado ser un material con capacidad osteogénica, biocompatibilidad, funciones inductivas y conductivas para la formación de tejido duro, presenta baja solubilidad y es altamente radiopaco, presenta una capacidad de selle superior a la amalgama, zOE (óxido de zinc y eugenol) e IRM, presenta un pH de 10,2 a 12,5 [11], similar al del hidróxido de cal- cio, causando también desnaturalización de proteínas, daño celular y destrucción de bacterias en el área en donde el material es colocado. Sin embargo, sus propiedades de biocompatibilidad hacen que este material tenga propiedades adicionales, como la formación de tejido duro similar al cemento, permite la adherencia osteoblástica y la regeneración ósea entre otras. El MTA también es utilizado como alternativa en ciertas condiciones clínicas además de la terapia pulpar, como reparación de perforaciones, reabsorciones y cirugía apical, entre otras, lo que hace al material ser una buena elección $[10,11,14,16-22]$.

El tratamiento de un diente con una lesión pulpar durante el periodo de maduración radicular se convierte en un desafío para el clínico, ya que, dependiendo de la vitalidad del órgano pulpar, la apexificación o la apexogénesis será el tratamiento de elección. El propósito de este reporte de caso es mostrar el cierre apical inducido mediante un procedimiento de apexogénesis con мтA de un molar inferior permanente.

\section{Reporte de caso}

Paciente de 7 años de edad, que acude al Servicio de Urgencias de la Facultad de Odontología de la Universidad Cooperativa de Colombia, sede Medellín, por presentar dolor ocasional; señala el diente 36; clínicamente presentaba caries extensa que comprometía espacio de cámara pulpar. En la radiografía periapical se evidenció el compromiso pulpar y el estadio del desarrollo radicular (estadio 8-9 nolla) (figura 1). A las pruebas clínicas respondió: percusión negativa, y a la prueba de sensibilidad al frío positiva ${ }^{\circ} 2$; el dolor cedió al retirar el estímulo. El diagnóstico del 36 fue de una pulpitis reversible con periápice sano. $\mathrm{Al}$ realizar la remoción de la caries se generó una exposición pulpar de gran tamaño, por lo cual se decidió realizar una pulpotomía y recubrimiento pulpar directo con MTA en la entrada de los conductos, con el fin de promover una apexogénesis a expensas de la pulpa vital remanente de cada uno de los conductos para promover el cierre apical de forma fisiológica. Se hizo el acceso con fresa redonda de diamante removiendo la caries por completo; el acceso convencional a la cavidad se realizó con una fresa de alta velocidad estéril utilizando e irrigando con abundante agua; se hizo la pulpotomía, y la desinfección de la cavidad se obtuvo con hipoclorito de sodio al 5,25\%; el sangrado fue controlado con una torunda de algodón estéril humedecida con solución 
anestésica lidocaína al 2\% con epinefrina 1: 80.000, con una presión suave por 2 a 5 minutos. Posterior a esto, el MTA (мTA Angelus ${ }^{\circledR}$ ) en polvo se mezcló con agua estéril de acuerdo con las instrucciones del fabricante, y la mezcla se colocó en el muñón pulpar en la entrada de los conductos radiculares con un espesor de $3 \mathrm{~mm}$ aproximadamente (figura 2) y se compactó con un condensador. Se dejó una torunda de algodón húmeda en la cámara pulpar para el fraguado del material, se selló la cavidad con cemento temporal. Posterior a esto, en la siguiente cita se eliminó el cemento temporal y el resto de la cavidad se llenó con ionómero de vidrio como selle coronal. Se realizó una cita de control a los ocho días, la paciente llegó asintomática y se hizo una restauración en resina. La paciente regresó a control un año después, y se comprobó que el diente estaba funcional, asintomático y con evidencia radiográfica de formación radicular normal en ambas raíces y el cierre apical completo (figura 3). Es importante tener en cuenta que las respuestas a pruebas de sensibilidad en este tipo de dientes con esta clase de tratamientos no son siempre posibles de determinar debido a que la pulpa cameral ya no está, y los conductos están sellados por el MTA, lo que aísla el estímulo y no es posible determinar el grado de respuesta; por tanto, el examen radiográfico y la sintomatología que pueda reportar o no el paciente son los determinantes en este caso del éxito o el fracaso del tratamiento.

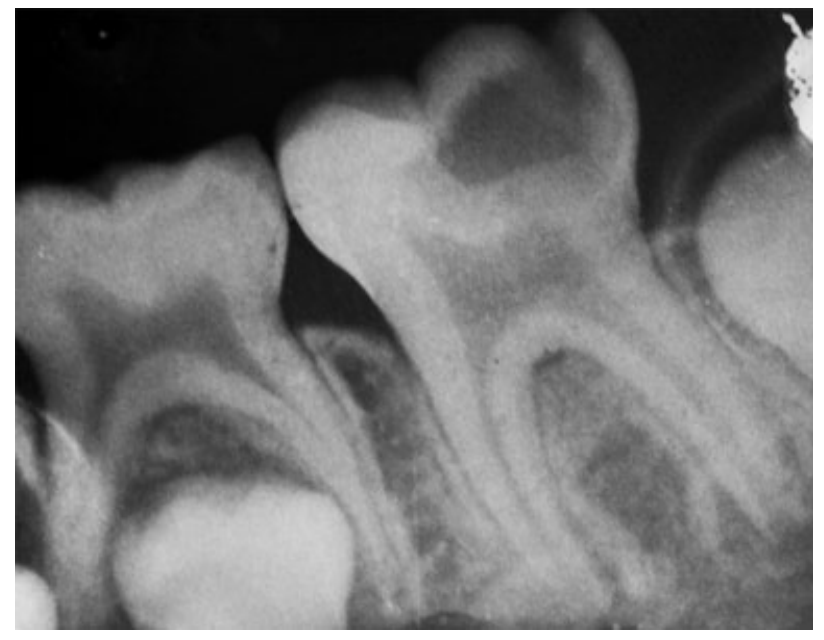

Figura 1. Radiografía de diagnóstico Fuente: elaboración propia

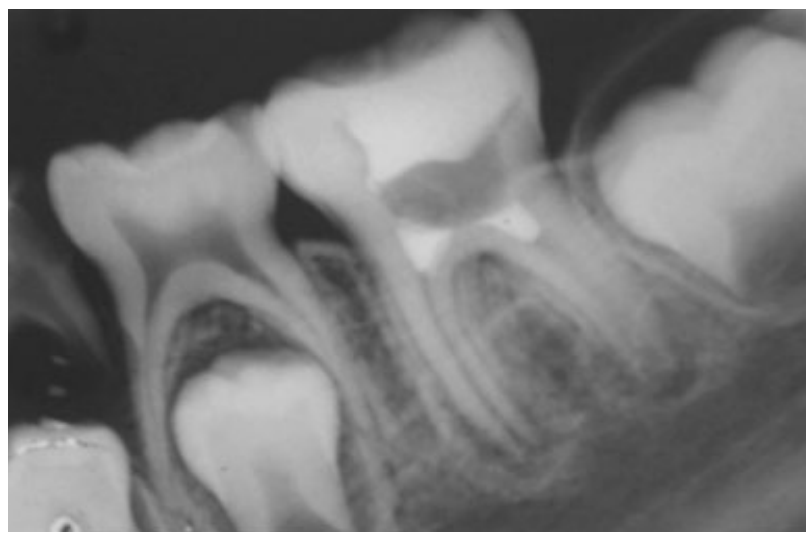

Figura 2. Radiografía de selle con MTA

Fuente: elaboración propia

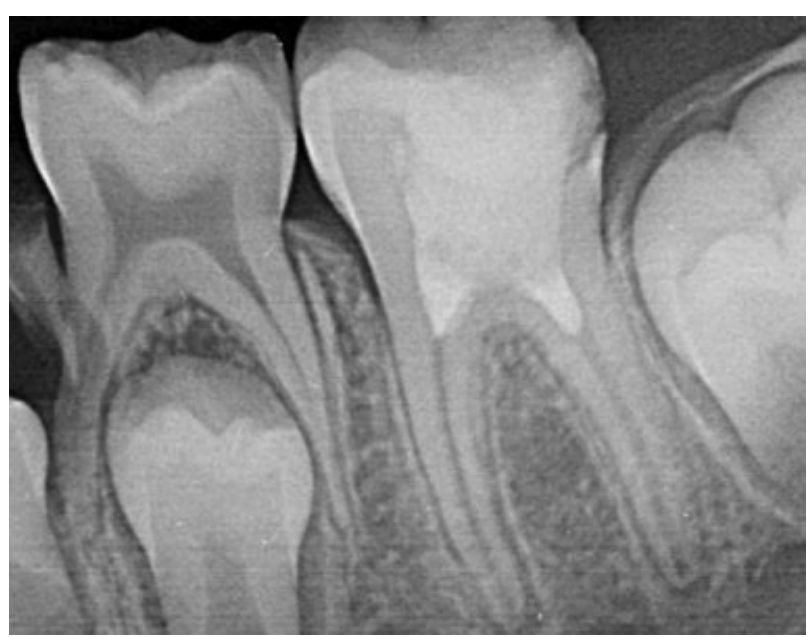

Figura 3. Radiografía control al año

Fuente: elaboración propia

\section{Discusión}

Muchos estudios in vitro e in vivo han demostrado que el MTA, por sus características, presenta buenas cualidades en cuanto a la prevención de filtración, biocompatibilidad, además de que no es reabsorbible, posee una buena resistencia a las fuerzas compresivas y capacidad de estimular la regeneración de tejidos como lo son la pulpa, además de los tejidos perirradiculares $[10,16,17,18,23]$. 
Patel señaló que con la terapia pulpar en dientes permanentes jóvenes expuestos a caries y con pulpitis, se puede evitar la necesidad de un tratamiento de conducto radicular, ya que en su reporte de caso mostró que el uso del MTA como material de recubrimiento pulpar directo con el fin de promover la continuación del desarrollo radicular normal de un diente con una pulpa afectada es factible. Clínica y radiográficamente demostró que el MTA se comportó de forma satisfactoria, y que puede ser considerado como un material eficaz y de elección para la terapia de una pulpa afectada pero vital, ya que se evidenció la terminación de la formación radicular [23].

Parviz describe el caso de una niña de ocho años de edad que fue tratada por fractura coronaria complicada de un incisivo central superior derecho debido a un accidente deportivo. El caso relata que realizaron la pulpectomía a los dientes con el fin de lograr una apexogénesis. El diente fue restaurado con una resina compuesta. El paciente fue controlado por un periodo de 10 años. Al inicio de los controles los dientes mostraron un desarrollo continuo y se observó formación de la porción apical de la raíz después del tratamiento pulpar vital; después de 10 años de seguimiento el diente desarrolló una necrosis pulpar y radiolucidez periapical. Después de la terapia de conducto radicular, la radiolucidez periapical cicatrizó [24]. Esto demuestra que aunque hay evidencia de éxito en este tipo de tratamientos, se hace necesario un control en tiempo considerable ya que existe la posibilidad de que en un momento determinado la pulpa remanente pueda necrosarse. Ahora bien, si después de lograrse el cierre apical, se presenta una necrosis del tejido pulpar, al estar los ápices completamente formados, se puede realizar el tratamiento endodóntico convencional sin necesidad de llevar a cabo una terapia de apexificación.

Sonoyama, en un estudio piloto, describe las características histológicas de la papila dental. El descubrimiento de células madre en esta estructura puede también explicar el fenómeno de apexogénesis mediante procesos de revitalizacion pulpar, en casos clínicos publicados de dientes inmaduros infectados. Se dice que estas células podrían sobrevivir a la infección por su proximidad con el tejido periapical, donde el abastecimiento de sangre es muy rico, a través del ápice completamente abierto [25]; esto nos demuestra el potencial de cicatrización que existe en las pulpas y los tejidos periapicales de pacientes jóvenes, lo que desempeña un papel fundamental en el éxito de una terapia pulpar.

Nosrat describe la apexogénesis de un segundo molar inferior derecho de una niña de 12 años de edad que fue remitida con una historia de dolor persistente y dolor a la masticación. Los exámenes clínicos y radiográficos revelaron caries coronal extensa y raíces con ápices abiertos; radiografías a los 3, 6 y 12 meses revelaron que el diente estaba funcional, sin signos o síntomas clínicos de patología pulpar [26]. El examen final confirmó el desarrollo radicular completo, así como la formación de un puente calcificado. En este reporte de caso evidenciamos que después de un año de evolución, la paciente continúa asintomática y sin signos de patología pulpar, y se observa que el desarrollo radicular se completó (figura 3). En el estudio de Omar y colaboradores, se demostró que el éxito de la apexogénesis con MTA vs. hidróxido de calcio obtuvo resultados satisfactorios, y se relata que de 15 dientes tratados con MTA, hubo éxito a los 12 meses de control en todos ellos comparado con 13 de 15 en el otro grupo. Sin embargo, recomienda realizar más estudios con poblaciones más grandes y tiempos de seguimientos más largos [27].

\section{Conclusiones}

La apexogénesis es un tratamiento muy útil para mantener dientes permanentes jóvenes con pulpa vital expuesta pero infectada, que aún no han terminado su desarrollo radicular, con el fin de promover de forma natural el cierre apical.

También se puede concluir que el MTA es un material de muy buenas características frente a otros como el hidróxido de calcio, lo cual lo hace una alternativa de elección para este tipo de tratamientos. En este reporte de caso se pudo observar que después de 12 meses postratamiento, el MTA mostró un excelente comportamiento para realizar pulpotomías mediante apexogénesis en dientes permanentes jóvenes con formación radicular incompleta. Sin embargo, es necesario que en este tipo de casos se realicen seguimientos y controles clínicos más extensos. 


\section{Referencias}

[1] Chaple Gil AM, Herrero Herrera L. Generalidades del agregado de trióxido mineral (MTA) y su aplicación en odontología. Revisión de Literatura. Acta Odontol Venez. 2007; 45(3): s. p.

[2] Villena Martínez H. Materiales utilizados en la terapia endodóntica de dientes primarios. En: Endodoncia pediátrica. Lima: Universidad Peruana Cayetano Heredia; 2005. p. 206.

[3] Hermann BW. Biologische Wurzelbehandlung. Frankfurt arn Main. En: Kramer W. Wurzelbehandlung. Deutsche Zahnarztliches Wochenschrift.1936; 38: 461-5.

[4] McDonald RE, Avery DR, Dean JA. Treatment of deep caries, vital pulp exposure and pulpless teeth. Dentistry for the child and adolescent. $8^{\mathrm{a}}$. ed. St. Louis: Mosby; 2004. p. 389-412.

[5] Anusavice KJ. Biocompatibility of dental materials. Phillip's science of dental materials. $10^{\text {th }}$. ed. Philadelphia: WB Saunders; 1996. p. 57-110.

[6] Andersen M, Lund A, Andreasen JO, Andreasen FM. In vitro solubility of human pulp tissue in calcium hydroxide and sodium hypochlorite. Endod Dent Traumatol. 1992; 8: 104-8.

[7] Barnes IE, Kidd EA. Disappearing Dycal. Br Dent J. 1979; 147: 111.

[8] Holland R, de Souza V, Nery MJ, Otoboni Filho JA, Bernabe PF, Dezan JE. Reaction of rat connective tissue to implanted dentin tubes filled with mineral trioxide aggregate or calcium hydroxide. J Endod. 1999; 25: 161-6.

[9] Torabinejad M, Chivian N. Clinical applications of mineral trioxide aggregate. J Endod. 1999; 25: 197-205.

[10] Torabinejad M, Hong CU, McDonald F, Pitt Ford TR. Physical and chemical properties of a new root-end filling material. J Endod. 1995; 21: 349-53.

[11] Maroto M, Barbería E, Vera V, García-Godoy F. Mineral trioxide aggregate as pulp dressing agent in pulpotomy treatment of primary molars: 42-month clinical study. Amer J Dent. 2007; 20(5): 283-6.

[12] Camilleri J, Pitt Ford TR. Mineral trioxide aggregate: a review of the constituents and biological properties of the material. Int Endod J. 2006; 39: 747-54.

[13] Schwartz RS, Mauger M, Clement DJ, Walker WA. Mineral trioxide aggregate: a new material for endodontics. J Am Dent Assoc. 1999; 130: 967-75.

[14] Dentsply Tulsa Dental. Material safety data sheet (MSDs). White ProRoot MTA root canal repair material. Okla: Tulsa Dental; 2001.
[15] Hakki SS, Bozkurt SB, Hakki EE, Belli S. Effects of mineral trioxide aggregate on cell survival, gene expresión associated with mineralized tissues, and biomineralization of cementoblasts. J Endod. 2009; 35: 513-9.

[16] Pitt Ford T, Torabinejad M, Abedi H, Kariyawasam S. Using mineral trioxide aggregate as a pulp-capping material. J Am Dent Assoc. 1996; 127: 1491-4.

[17] Sluyk SR, Moon PC, Hartwell GR. Evaluation of setting properties and retention characteristics of mineral trioxide aggregate when used as a furcation perforation repair material. J Endod. 1998; 24: 768-71.

[18] Holland R, Souza V, Nery MJ. Calcium salts deposition in rat connective tissue after the implantation of calcium hydroxide-containing sealers. J Endod. 2002; 28: 173-6.

[19] Tamburic SD, Vuleta GM, Ognjanovic JM. In vitro release of calcium and hydroxyl ions from two types of calcium hydroxide preparation. Int Endod J. 1993; 26: 125-30.

[20] Favieri A, Campos LC, Burity VH, Cecilia MS, Abad EDC. Use of biomaterials in periradicular surgery: a case report. J Endod. 2008; 34: 490-4.

[21] Do Nascimento C, Issa JPM, Iyomasa MM, Regalo $\mathrm{SCH}$, Siessere S, Pitol DL et al. Bone repair using mineral trioxide aggregate combined to a material carrier, associated or not with calcium hydroxide in bone defects. Micron. 2008; 39: 868-74.

[22] Banchs F, Trope M. Revascularization of immature permanent teeth with apical periodontitis: new treatment protocol. J Endod. 2004; 30: 196-200.

[23] Patel R, Cohenca N. Maturogenesis of a cariously exposed immature permanent tooth using MTA for direct pulp capping: acase report. Dent Traumatol. 2006; 22: 238-333.

[24] Parviz A, Masoud P. The importance of long time follow-up after vital pulp therapy: A case report. Iran Endod J. 2008; 3: 90-2.

[25] Sonoyama W, Liu Y, Yamaza T, Tuan RS, Wang S, Shi S, Huang GT. Characterization of de apical papila and is residing stem cells from human immature permanente teeth: a pilot study. J Endod. 2008; 34: 166-71.

[26] Nosrat A, Asgary S. Apexogenesis of a symptomatic molar with calcium enriched mixture. Int Endod J. 2010; 43: 940-4.

[27] Omar AS, David R. A. Comparison of mineral trioxide .aggregate and calcium hydroxide as pulpotomy agents in young permanent teeth (apexogenesis). Department of Pediatric Dentistry and Public Health, Faculty of Dentistry, Alexandria University, Alexandria, Egypt. Pediatric Dent. 2006; 28: 399-404. 